M. Kindermann

G. Fröhlig

\title{
Frequenzadaptive Schrittmachertherapie bei chronotroper Inkompetenz - Therapie
}

\section{Rate adaptive pacing in patients with chronotropic incompetence - Therapy}

\section{Leistungssteigerung durch frequenzvariable Stimulation}

Dass Wiederherstellung der Chronotropie leistungssteigernd wirkt, sei exemplarisch an einer Studie gezeigt, in der 27 frequenzinkompetente Patienten im Durchschnittsalter von 60 Jahren mittels symptomlimitierter Spiroergometrie untersucht und mit einem 41-köpfigen, altersentsprechenden Normalkollektiv verglichen wurden (4).

Die Befunde zeigt Tab.1. Parallel zur Anhebung der Herzfrequenz auf Werte der Vergleichsgruppe bewirkt die frequenzvariable Stimulation des Schrittmachers (VVIR) an der ventilatorisch anaeroben Schwelle eine signifikant höhere Sauerstoffaufnahme und Leistung gegenüber VVI, bleibt mit 79\% der Kontrollwerte aber deutlich hinter den Herzgesunden zurück.

Das Beispiel ist gewählt, weil die Analyse sich nicht wie üblich auf die Sauerstoffaufnahme $\left(\mathrm{VO}_{2}\right)$ bei Maximalbelastung oder anaerober Schwelle (AT) beschränkt, sondern zusätzlich $\mathrm{O}_{2}$-Aufnahme und Leistung miteinander in Beziehung setzt. Bei kontinuierlicher Laststeigerung unterhalb der AT (wie sie in der Studie vorgenommen wurde) oder bei Steady-state-Bedingungen kann der „, $\mathrm{VO}_{2}$-Leistungsindex“ $\left(\mathrm{dVO}_{2} / \mathrm{dWR}\right)$ als Maß dafür gelten, wie viel Sauerstoff das Transportsystem des Körpers an die arbeitende Zelle liefern kann, um eine vorgegebene Leistung zu ermöglichen; er ist damit ein geeigneter Parameter zur Beurteilung der Ausdauerleistungsfähigkeit. Im Beispiel zeigt dieser Index eine Steigerung um 29\% zwischen festfrequenter und frequenzadaptiver Stimulation und lässt damit keinen Unterschied zum Normalkollektiv mehr erkennen (Tab.1).

In der Schrittmacherliteratur nicht ungewöhnlich sind Befunde, nach denen Akut-Effekte der Stimulation im chronischen Verlauf weniger ausgeprägt sind oder gänzlich verschwinden (13),
Tab.1 Herzfrequenz (HF), Sauerstoffaufnahme $\left(\mathrm{VO}_{2}\right)$ und Leistung (W) an der anaeroben Schwelle (AT), sowie $\mathrm{VO}_{2}$-Leistungsindex $\left(\mathrm{dVO}_{2} / \mathrm{dWR}\right.$ ) unter AT bei 27 chronotrop inkompetenten Patienten und 41 normalen Probanden. Unter frequenzadaptiver Stimulation bleibt die $\mathrm{VO}_{2}$-AT subnormal, der Leistungsindex jedoch differiert nicht zwischen Patienten mit frequenzadaptiver Stimulation und Normalkollektiv. (n bitte die Kernaussage dieser Tabelle in einem Satz zusammenfassen)

\begin{tabular}{|c|c|c|c|c|c|}
\hline$H F[1 / \min ]$ & $\begin{array}{l}V V I \\
75 \pm 9\end{array}$ & $\begin{array}{l}\text { VVIR } \\
113 \pm 21\end{array}$ & $\begin{array}{l}p \\
a\end{array}$ & $\begin{array}{l}\text { Normal } \\
106 \pm 16\end{array}$ & $\begin{array}{l}p \\
a\end{array}$ \\
\hline$V_{2}-A T[\mathrm{ml} / \mathrm{kg} / \mathrm{min}]$ & $9,3 \pm 3,4$ & $10,9 \pm 4,3$ & $x$ & $14,7 \pm 2,8$ & $a, b$ \\
\hline W-AT [Watt] & $52 \pm 20$ & $65 \pm 24$ & $\mathrm{a}$ & $79 \pm 22$ & $a, c$ \\
\hline $\begin{array}{l}d \mathrm{VO}_{2} / \mathrm{dWR}[\mathrm{ml} / \mathrm{min} / \\
\text { Watt] }\end{array}$ & $7,9 \pm 2,3$ & $10,2 \pm 2,4$ & $a$ & $10,3 \pm 1,4$ & $\mathrm{a}$ \\
\hline \multicolumn{6}{|c|}{$\begin{array}{l}\text { WV: festfrequente Stimulation mit 70/min (höhere Ist-Frequenz infolge inter- } \\
\text { mittierender Spontanrhythmen), VVIR: frequenzadaptierter Modus, a: } p<0,001 \\
\text { zu WVI, b: } p<0,001 \text { zu VVIR, c: } p<0,05 \text { zu VVIR, x: keine Signifikanz in Original- } \\
\text { tabelle angegeben, nach Text wie a (nach (4)) }\end{array}$} \\
\hline
\end{tabular}

weil Mechanismen zur Kompensation einer gestörten Hämodynamik nach deren Korrektur nicht mehr benötigt und deshalb inaktiviert werden. Während die hämodynamische Überlegenheit der vorhofgesteuerten (DDD-/VDD-) gegenüber der festfrequenten Ventrikelstimulation (VVI) im Langzeitverlauf belegt ist $(2,6,11,14,15)$, sind die Daten zum chronischen Effekt der (Vorhof-unabhängigen) frequenzvariablen Schrittmacherbehandlung weniger eindeutig. So wird einerseits nach je 10 -wöchiger Laufzeit zwischen frequenzfixierter und -variabler Schrittmacherbehandlung kein signifikanter Unterschied mehr für den $\mathrm{VO}_{2}$-Leistungsindex gefunden (Tab.2) (5).

Andererseits werden Befunde mitgeteilt, nach denen sowohl die maximale wie auch die Sauerstoffaufnahme an der anaeroben Schwelle 
nach 4-wöchiger Stimulation im frequenzadaptiven Modus gegenüber festfrequenter Stimulation erhöht bleiben (Tab.3) (9).

In einer echokardiographischen Studie an 13 Patienten (davon zehn mit AV-Block II.-III. Grades und sechs bereits zuvor mit einem VVISchrittmacher ohne Frequenzantwort behandelt) zeigt sich binnen 6 Monaten als wesentlicher Effekt frequenzvariabler Stimulation eine Minderung des linksventrikulären enddiastolischen Querdurchmessers von $65 \pm 6$ auf $56 \pm 4 \mathrm{~mm}$. Weil der systolische Durchmesser unverändert bleibt, sinkt die Verkürzungsfraktion in Ruhe von $33 \pm 4$ auf $27 \pm 3 \%$ ( $p<0,05)$, was nicht zwingend als systolische Funktionsminderung, sondern auch als Zunahme der Kontraktionsreserve unter Belastung interpretiert werden kann (7).

\section{Indikation zur frequenzadaptiven Stimulation}

Anders als die amerikanischen (8) begründen die Leitlinien der Deutschen Gesellschaft für Kardiologie keine Stimulationsindikation allein aus symptomatischer Frequenzinkompetenz (12). Allerdings ist die Implantation eines frequenzadaptiven Systems mit der Symptomatik und der jeweiligen Reizbildungs- oder Leitungsstörung zu rechtfertigen, welche den Frequenzanstieg behindert. Ist bereits ein Schrittmacher implantiert, so geht es darum, ob die aggregatseitig vorgehaltene Frequenzmodulation (R) aktiviert oder - falls nicht verfügbar - ein vorzeitiger Systemtausch vorgenommen werden soll. Die Indikation ist gegeben, wenn

- der Patient symptomatisch ist,

dies zu wesentlichen Teilen der mangelnden Frequenzsteigerung zugeschrieben werden muss (siehe Abschnitt „Praktische Diagnostik“) und

auf eine eventuell bradykardisierende Medikation nicht verzichtet werden kann.

\section{Technisches Rüstzeug}

Bis auf das Weglassen verzichtbarer Pharmaka ist keine medikamentöse Maßnahme geeignet, eine ausreichende Chronotropie (wieder) herzustellen. Antibradykard eingesetzte Sympathomimetika oder Vagolytika haben den Nachteil, dass sie auch die Ruhefrequenz anheben, wenn sie unter Belastung effektiv sein sollen. Weil dies auf Dauer nicht vertragen wird, bleibt nur die Schrittmacherbehandlung.

Ist die mangelnde Chronotropie Folge einer AV-Blockierung, so stellt die Anbindung der Kammern an die physiologische Frequenzregulation der Vorhöfe die Therapie der Wahl dar. Technisch wird dies mit einem AV-sequenziellen System ohne (VDD) oder mit der Option zur Vorhofstimulation (DDD) realisiert. Bei krankem oder ausgefallenem Sinusknoten ist ein sensorgesteuerter Schrittmacher erforderlich, der je nach Funktion des AV-Knotens als Ein- (AAIR) bzw. Zweikammer-System (DDDR) ausgeführt werden kann. Persistierendes Vorhofflimmern mit bradykarder Kammerantwort unter Belastung wird mit einem VVIR-Schrittmacher behandelt.

Die Frequenzvariation basiert auf belastungsinduzierten Signalen, die von geeigneten Sensoren aufgenommen, meist gemittelt und mit einem gleitenden Referenzwert verglichen wer-
Tab.2 Steigung der linearen Regression zwischen Herzfrequenz und Ventilation (dHF/dVE), Herzfrequenz und Sauerstoffaufnahme $\left(\mathrm{dHF} / \mathrm{dVO}_{2}\right)$, sowie $\mathrm{VO}_{2}$-Leistungsindex $\left(\mathrm{dVO}_{2} / \mathrm{dWR}\right)$ bei festfrequenter ( $\mathrm{VVI})$ und Atemminutenvolumen-kontrollierter Stimulation (VVIR) akut und nach 10-wöchigem Betrieb im jeweiligen Modus ( $n=14$; chronotrope Inkompetenz; keine Angaben über patienteneigenen Rhythmus). Für den Leistungsindex lässt sich im chronischen Verlauf kein Unterschied zwischen VVI und VVIR finden.

\begin{tabular}{|c|c|c|c|c|}
\hline & $\begin{array}{l}\text { VVI } \\
\text { akut }\end{array}$ & chronisch & $\begin{array}{l}\text { VVIR } \\
\text { akut }\end{array}$ & chronisch \\
\hline$d H F / d V E[b p m / l / m i n]$ & $0,1 \pm 0,2$ & $0,2 \pm 0,3$ & $\begin{array}{l}3,3 \pm 1,1 \\
\#\end{array}$ & $\begin{array}{l}3,4 \pm 1,0 \\
\#\end{array}$ \\
\hline $\begin{array}{l}\mathrm{dHF} / \mathrm{dVO} \mathrm{O}_{2}[\mathrm{bpm} / \mathrm{ml} / \\
\left.\mathrm{kg}{ }^{*} \mathrm{~min}\right]\end{array}$ & $1,1 \pm 1,1$ & $1,4 \pm 1,4$ & $\begin{array}{l}5,9 \pm 2,5 \\
\#\end{array}$ & $\begin{array}{l}4,9 \pm 1,6 \\
\left.\#\right|_{+} ^{+}\end{array}$ \\
\hline $\begin{array}{l}d \mathrm{dVO}_{2} / \mathrm{dWR}[\mathrm{ml} / \mathrm{min} / \\
\text { Watt] }\end{array}$ & $7,8 \pm 1,8$ & $8,8 \pm 2,5$ & $\begin{array}{l}10,9 \pm 3,4 \\
\#\end{array}$ & $\begin{array}{l}9,0 \pm 2,1 \\
\mathrm{~ns} /+\end{array}$ \\
\hline \multicolumn{5}{|c|}{$\begin{array}{l}\text { \#: } p<0,01, \text { VVI vs. VVIR, +: } p<0,05, \text { akut vs. Chronisch, ns: nicht signifikant, VVI } \\
\text { vs. VVIR (nach (5)). }\end{array}$} \\
\hline
\end{tabular}

Tab.3: Frequenzvariable VVI-Stimulation bei 14 Patienten mit AVBlock ( $n=11$ ) oder sinuatrialer Störung $(n=3)$ durch Aggregate mit Atemvolumen- $(n=9)$, QT- $(n=1)$ oder Dual-sensorSteuerung (Aktivitäts- und QT-Steuerung). Steigerung des jeweiligen Parameters gegenüber VVI in Prozent. (nach (9)).

\begin{tabular}{|c|c|c|c|c|c|c|}
\hline & $H F_{\max }$ & $H F_{A T}$ & $V O_{2 \max }$ & $V O_{2 A T}$ & $W_{\max }$ & $W_{A T}$ \\
\hline akut & $\begin{array}{l}41 \pm 21 \\
\#\end{array}$ & $\begin{array}{l}34 \pm 20 \\
\#\end{array}$ & $\begin{array}{l}23 \pm 22 \\
\#\end{array}$ & $\begin{array}{l}32 \pm 28 \\
\#\end{array}$ & $\begin{array}{l}18 \pm 21 \\
\#\end{array}$ & $\begin{array}{l}33 \pm 27 \\
\#\end{array}$ \\
\hline chronisch & - & - & $\begin{array}{l}36 \pm 41 \\
p<0,01\end{array}$ & $\begin{array}{l}29 \pm 42 \\
p<0,05\end{array}$ & $\begin{array}{l}17 \pm 19 \\
p<0,01\end{array}$ & $\begin{array}{l}19 \pm 27 \\
n s\end{array}$ \\
\hline \multicolumn{7}{|c|}{$\begin{array}{l}H F_{\text {max }}: \text { Maximale Herzfrequenz, } H F_{A T}: \text { Herzfrequenz an der anaeroben Schwelle, } \\
V_{2} O_{2 m a x} \text { Maximale Sauerstoffaufnahme, } V_{2 A T}: \text { Sauerstoffaufnahme an der } \\
\text { anaeroben Schwelle, } W_{\text {max }}: \text { Maximale Leistung, } W_{A T} \text { : Leistung an der anaeroben } \\
\text { Schwelle, \#: signifikant (p nicht spezifiziert), ns: nicht signifikant }\end{array}$} \\
\hline
\end{tabular}

den. Derzeit verfügbare Messsysteme sind in Tab.4 aufgelistet und in ihren wesentlichen Eigenschaften klassifiziert. Details sind der einschlägigen Schrittmacherliteratur zu entnehmen. Positive Eigenschaften eines frequenzvariablen Systems sind: schnelle Reaktion, lastproportionale Frequenzänderung, niedrige Störanfälligkeit. Die Systeme sollten mit (unipolaren) Standardelektroden und ohne Zwangsstimulation in der Kammer zu betreiben sein, welche mit unphysiologischer Erregungsausbreitung die linksventrikuläre Funktion kompromittieren kann. Regeltechnisch optimal wäre die Fähigkeit der Systeme zur negativen Rückkopplung, die physiologisch inadäquate Frequenzsteigerungen verhindern könnte. Bis auf die Sensoren, die sympathikusabhängige Parameter erfassen (QT, PEA, CLS), ist diese Forderung (auch in Ansätzen) nicht erfüllt.

Tab.4 lässt erkennen, dass bis auf den Sinusknoten, dessen Frequenzaktivität mittelbar im Vorhof abgegriffen werden kann, kein Sensor die optimale Merkmalkombination aufweist. Für den isolierten AV-Block ist deshalb die Vorhofsteuerung eines DDD- oder VDD-Schrittmachersystems die optimale Therapie. Sonst versuchen so genannte „Zweisensor-Systeme“ die Nachteile des einen durch die Vorzüge des anderen Messprinzips auszugleichen. Typisch ist 
Tab.4 Wichtige Eigenschaften der gegenwärtig kommerziell erhältlichen Ein-Sensoren-Systeme.

\begin{tabular}{|c|c|c|c|c|c|c|c|}
\hline Sensor & Phys & $\operatorname{Reg}$ & Reak & Stör & Sonde & V-Pace & Besonderheiten \\
\hline Sinusknoten & + & + & $S$ & $\mathrm{~N}$ & A & - & als Sensor für DDD-System geeignet \\
\hline Aktivität & - & - & $\mathrm{S}$ & $\mathrm{H}$ & A & - & ext. Störpotential mit Akzelerometer $\downarrow$ \\
\hline$A M V$ & + & - & M & M & B & - & hohe Frequenz auch nach Belastung \\
\hline$Q T$ & + & $(+/-)$ & $\mathrm{L}$ & $\mathrm{H}$ & A & $(+)$ & starke Medikamenteneinflüsse \\
\hline PEA & + & + & $?$ & $?$ & $\mathrm{P}$ & - & \\
\hline
\end{tabular}

Aktivität: Körpervibrationen, richtungssensitive Beschleunigung; AMV: transthorakale Impedanzmodulation als Äquivalent des Atemminutenvolumens, QT: stimuliertes endokardiales QT-Intervall, PEA: mittels Piezoquarz an der ventrikulären Sondenspitze aufgenommene „Peak Endocardial Acceleration“, CLS: „Closed Loop Stimulation“ (nutzt lokale Impedanzänderungen, die kontraktionsabhängige Änderungen des Kammervolumens im Umkreis der Sondenspitze wiederspiegeln).

Phys: Last-proportionale Reaktion auf physiologisches Signal, Reg: sympathikusvermittelte negative Rückkopplung, Reak: Reaktionsgeschwindigkeit, Stör: externe Störanfälligkeit, Sonde: Sondenspezifikation, V-Pace: Signalmessung setzt stimulierte ventrikuläre Depolarisation voraus.

+: Merkmal vorhanden, -: Merkmal nicht vorhanden, ?: durch Literatur nicht ausreichend belegt, S: schnell, M: mittel, L: langsam, N: niedrig, H: hoch, A: alle Modelle, B: bipolar, P: herstellerspezifisch)

die Kombination aus schnell reagierendem, aber unphysiologisch arbeitendem Bewegungssensor und langsamer, aber lastproportionaler QT- oder Atemminutenvolumen-Steuerung. Der Input beider Sensoren wird durch Algorithmen so verknüpft, dass beide einander auf Plausibilität prüfen („Sensor Cross Checking“) oder in der Frequenzantwort beeinflussen können („Sensor Blending“).

\section{Programmier-Empfehlungen}

Die zunehmende Komplexität frequenzadaptiver Systeme lässt die manuelle Einstellung von Sensor-Reaktionsschwellen, Frequenzkennung, prinzipspezifischen Parametern und letztlich der Maximalfrequenz zum aussichtslosen Unterfangen werden, zumal der in fast allen Systemen integrierte Bewegungssensor am Fahrradergometer kaum reagiert und für die Praxis nicht getestet werden kann. Lösungsansatz ist eine Automatik, welche das System so optimiert, dass es in definierten Zeiträumen (z.B. einmal täglich) die Bandbreite zwischen unterer und oberer Grenzfrequenz ausnutzt und - sofern das mit der aktuellen Einstellung nicht gelingt - die Kennung anpasst. Das grob skizzierte Prinzip wird von Herstellerseite vielfach verfeinert. In der Verantwortung des Arztes bleibt es zu entscheiden, von welcher Herzfrequenz der Patient profitiert oder möglicherweise Schaden nimmt. Das medizinische Problem ist also das Frequenzlimit.

Beim symptomatischen Koronarkranken ist das die Anginaschwelle (siehe „Der konkrete Fall“). Klinisch weniger eindeutig zu entscheiden ist dies beim Vorliegen einer myokardialen Relaxations- oder Compliancestörung, die hohe Frequenzen mit Verkürzung der Diastolendauer nicht verträgt. Bei endgradiger Pumpschwäche des Herzens gilt es, die Chronotropie so einzustellen, dass entlang steiler Frequenzkennung Leistungsreserven mobilisiert werden, bevor die inverse Kraft-Frequenzkopplung $(1,3)$ die Auswurfleistung einbrechen lässt.

Generelle Empfehlung ist deshalb, bei organisch Herzkranken die Maximalfrequenz des Schrittmachers bei $100-110$ /min zu begrenzen. Im Einzelfall kann das individuelle Limit spiroergometrisch daran erkannt werden, dass trotz Frequenzsteigerung die Sauerstoffaufnahme nicht mehr zunimmt („Plateau“) oder gar abfällt (Abb.1) (10).
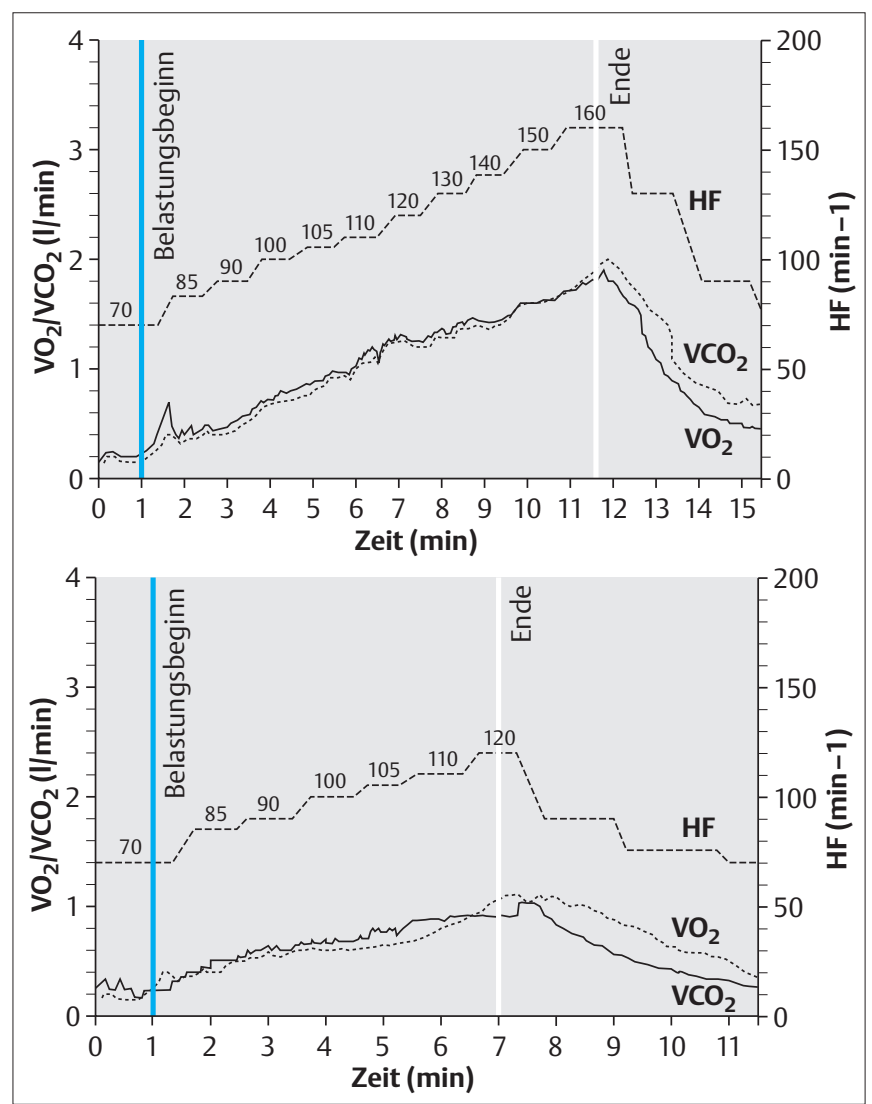

Abb.1 Spiroergometrie mit Aufzeichnung der Herzfrequenz (HF), der Sauerstoffaufnahme $\left(\mathrm{VO}_{2}\right)$ und Kohlendioxidabgabe $\left(\mathrm{VCO}_{2}\right)$. Links: Proband ohne organische Herzkrankheit, bei dem die Sauerstoffaufnahme bis zur Maximalbelastung mit der Frequenz korreliert. Rechts: Patient mit DCM (Ejektionsfraktion $30 \%$ ) und $\mathrm{VO}_{2}$-Plateau bei einer Herzfrequenz von 110/min (modifiziert nach (10)).

kurzgefasst: Nach den amerikanischen, nicht jedoch nach den deutschen Leitlinien zur Schrittmachertherapie stellt die symptomatische chronotrope Inkompetenz eine Klasse-I-Indikation zur Schrittmacherbehandlung dar. Die Programmierung der Frequenzadaption setzt neben der Kenntnis des jeweiligen Schrittmachersystems immer auch die Kenntnis der individuellen kardia- 


\section{len Grunderkrankung voraus. Bei bedeutsamer organi- scher Herzkrankheit sollte das Frequenzlimit des Schrittmachers niedrig (100-110/min) gewählt werden.}

eratur

Alpert NR, Leavitt BJ, Ittleman FP, Hasenfuss G, Pieske B, Mulieri LA. A mechanistic analysis of the force-frequency relation in non-failing and progressively failing human myocardium. Basic Res Cardiol 1998; 93: 23-32 Bergbauer M, Sabin G. Hämodynamische Langzeitresultate der bifokalen schrittmacher-Stimulation. Dtsch med Wochenschr 1983; 108: 545-549 Böhm M, La Rosée K, Schmidt U, Schulz C, Schwinger RHG, Erdmann E. Force-frequency relationship and inotropic stimulation in the nonfailing and failing human myocardium: implications for the medical treatment of heart failure. Clin Investig 1992; 70: 421-425

${ }^{4}$ Epperlein S, Treese N, Stegmaier A, Coutinho M, Meyer J. Der V02-Leistungsindex zur Beurteilung der kardiopulmonalen Leistungsfähigkeit unter atemminutenvolumengesteuerter VVI-R-Stimulation. Z Kardiol 1994; 83: 343-350

${ }^{5}$ Epperlein S, Treese N, Herrig I, Werneyer A, Zegelmann M, Sammer P, Meyer J. Car-diopulmonary exercise capacity in single chamber rate adaptive pacing Can the acute improvement be maintained? (abst). PACE 1993; 16: 1918

${ }^{6}$ Faerestrand S, Ohm OJ. A time-related study of the hemodynamic benefit of atrioventricular synchronous pacing evaluated by Doppler echocardiography. PACE 1985; 8: 838-848

7 Faerestrand $\mathrm{S}$, Ohm OJ. A time-related study by Doppler and M-mode echocardiography of hemodynamics, heart rate, and AV valvular function during activity-sensing rate-responsive ventricular pacing. PACE 1987; 10: 507-518

${ }^{8}$ Gregoratos G, Cheitlin MD, Conill A, Epstein AE, Fellows C, Ferguson TB, Freedman RA, Hlatsky MA, Naccarelli GV, Saksena S, Schlant RC, Silka MJ. ACC/AHA guidelines for Implantation of cardiac pacemakers and antiarrhythmia devices. Circulation 1998; 97: 1325-1335

9 Haltern G, Sigmund M, Ochs JG, Kempa L, Firnich G, Hanrath P. Chronic effect of rate adaptive pacing on exercise capacity (abst.). PACE 1993; 16: 1921

10 Kindermann M, Schwaab B, Finkler N, Schaller S, Böhm M, Fröhlig G. Defining the optimum upper heart rate limit during exercise - A study in pacemaker patients with heart failure. Eur Heart J (in press)

11 Kruse I, Amman K, Conradson TB, Ryden L. A comparison of the acute and long-term hemodynamic effects of ventricular inhibited and atrial synchronous ventricular inhibited pacing. Circulation 1982; 65: 846-855

12 Lemke B, Fischer W, Schulten K. Richtlinien zur Herzschrittmachertherapie. Z Kardiol 1996; 85: 611-628

13 Nager F, Bühlmann A, Schaub F. Klinische und hämodynamische Befunde beim totalen AV-Block nach Implantation elektrischer Schrittmacher. Helvetica Medica Acta 1966; 33: 240-276

${ }^{14}$ Sutton R, Perrins EJ, Morley C, Chan SL. Sustained improvement in exercise tolerance following physiological cardiac pacing. Eur Heart J 1983; 4: 781-785

15 Yee R, Benditt DG, Kostuk WJ, Ko PT, Purves P, Klein GJ. Comparative functional effects of chronic ventricular demand and atrial synchronous ventricular inhibited pacing. PACE 1984; 7: 23-28 